\title{
Selection of patients with Tourette syndrome for deep brain stimulation surgery
}

\author{
Mauro Porta ${ }^{\mathrm{a}, *}$, Andrea E. Cavanna ${ }^{\mathrm{b}, \mathrm{c}}$, Edvin Zekaj $^{\mathrm{d}}$, Francesca D’Adda $^{\mathrm{a}}$ and Domenico Servello ${ }^{\mathrm{d}}$ \\ ${ }^{a}$ Tourette Centre, IRCCS Galeazzi Hospital, Milan, Italy \\ ${ }^{\mathrm{b}}$ The Michael Trimble Neuropsychiatry Research Group, Department of Neuropsychiatry, BSMHFT and University \\ of Birmingham, Birmingham, UK

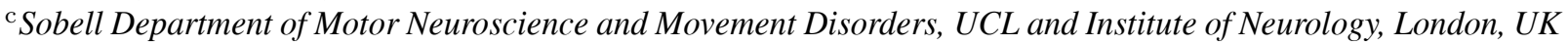 \\ ${ }^{\mathrm{d}}$ Functional Neurosurgery Unit, IRCCS Galeazzi Hospital, Milan, Italy
}

\begin{abstract}
Deep brain stimulation (DBS) is an emerging therapeutic option for severe resistant Tourette syndrome (TS). To date, about 100 cases have been reported in the scientific literature. Different clinical guidelines have been proposed for this procedure from both USA and European centres. A number of issues remain unresolved, mainly in relation to eligibility criteria of patients with TS. We highlight the need for a comprehensive assessment of associated co-morbidities, which are not considered integral part of the syndrome and are not sufficiently evaluated in relation to DBS. The concept of refractoriness, the minimum age of candidates, and the optimal targets for DBS are also controversial.
\end{abstract}

Keywords: Deep brain stimulation, Tourette syndrome, tics, co-morbidities, selection criteria

\section{Introduction}

Tourette syndrome (TS) is a chronic neurodevelopmental disorder defined by the presence of multiple motor tics and at least one phonic tic [1]. It is estimated that about $90 \%$ of patients have at least one co-morbid behavioural symptom, such as attention deficit hyperactivity disorder (ADHD), obsessive-compulsive disorder (OCD), self-injurious behaviour (SIB), impulse dyscontrol and affective dysregulation [2]. Overall, the course of tic symptoms is relatively favourable over time: up to $50 \%$ of patients with onset of tic disorder before age 10 experience a significant decrease in tic severity during adolescence [3]. However, severe TS can have devastating effects on perceived well being and relationships. Behavioural strategies, in addition to both standard and innovative pharmacological therapies, are the main treatment interventions for patients

*Corresponding author: Mauro Porta, Tourette Centre, IRCCS Galeazzi Hospital, Via R. Galeazzi 4, 20161 Milan, Italy. Tel.: +39 02/66214933; Fax: +39 02/66214916; E-mail: portamilano@ libero.it. who continue to experience severe symptoms with significant social impairment.

When patients are refractory to conservative treatments, deep brain stimulation (DBS) could be considered as an emerging therapeutic option. Over the last few decades, DBS has been demonstrated to be a safe and efficacious procedure in different movement disorders, including Parkinson disease, essential tremor and dystonia [4]. More recently, DBS has also been used to treat patients with severe and refractory psychiatric disorders, such as OCD and depression [5].

The rationale for choosing specific targets for DBS to treat patients with severe and refractory TS was initially based on the effects of historical procedures of ablative surgery, which are no longer ethically acceptable [6]. The results of preliminary reports on DBS in TS are encouraging, even though many aspects have yet to be better clarified, in primis the issue of patient selection [7].

\section{Assessment}

Candidates to DBS in TS should undergo a comprehensive neurological, neuropsychiatric and neuropsy- 
chological pre-surgical assessment, to be carried out by a multidisciplinary team with clinical experience in TS and in collaboration with functional neurosurgeons. Detailed clinical assessment guidelines of tic disorders and common co-morbidities are discussed in the recently published guidelines from the European Society for the Study of Tourette Syndrome (ESSTS) [8]. Patients should be diagnosed according to DSM IV-TR criteria and the diagnosis should be validated by instruments such as the TS Diagnostic Confidence Index. With regards to the clinical picture, the tic symptoms should represent the primary problem significantly affecting Quality of Life (QoL).

Tic severity should be evaluated by means of direct observation (e.g. videorecording) and clinical scales, such as the Yale Global Tic Severity Scale (YGTSS). A YGTSS total tic severity score above 35/50 during repeated assessments for at least six months is a reliable indicator of a degree of tic severity prompting consideration of DBS.

The large majority of patients with TS have comorbid psychopathology, including attention-deficit and hyperactivity disorder (ADHD), obsessivecompulsive disorders (OCD), anger control problems, mood disorders, conduct and oppositional defiant disorders. Co-morbidities have to be evaluated using specific scales: for example, the Yale-Brown Obsessive Compulsive Scale (YBOCS) has often been used for evaluation of obsessive compulsive behaviours; the StateTrait Anxiety Inventory (STAI) assesses patients' state and trait anxiety, and the Beck Depression Inventory (BDI) reliably measures depression. It should always be considered that co-morbidity can represent the main reason to seek help in patients with TS. The disease-specific health-related quality of life scale for TS (GTS-QOL) developed by Cavanna et al. [9] highlights the relevance of the patient's subjective point of view. This scale comprises 27 items which examine four components of quality of life: the psychological domain, the physical/activities of daily living (ADL) domain, the obsessive-compulsive domain and the cognitive domain. Importantly, this scale can adequately and specifically evaluate aspects of social impairment which often motivate DBS in refractory patients. The GTS-QOL also captures other aspects of the TS-related impact on life. Self injurious behaviour (SIB) is frequently present in the context of TS. For example, SIB of different severity was reported in $46.1 \%$ of patients from a clinical sample of 639 subjects [10]. Patients with life-threatening tics are classified as having "malignant TS". Jankovic et al. [11] in their experience reported that $5 \%$ of patients with TS can present malignant TS, often in association with severe obsessivecompulsive symptomatology. Finally, the GTS-QOL can prove useful both as baseline and outcome measure, also in the longer term. For instance, a recent study showed that young patients with severe tics associated with characteristic premonitory urges and a family history of tics disorders appear to be at higher risk for poorer quality of life as adults [12].

Both environmental and psychosocial stressors have to be evaluated as factors aggravating clinical symptoms. Surgical candidates should experience tics associated with a significant functional impairment.

"TS by history" has to be carefully evaluated by asking patients, parents and caregivers. These data can be highly informative, although the value of these parameters is not yet formally validated. At least familiar, environmental and economic issues have to be carefully examined as they are important factors that may influence patient compliance. Analysis of the phenomenological aspects of TS according to Jankovic [13] seems essential to choose targets for DBS; videotaping according to Kompoliti and Goetz [14] and accelerometry [15] can be used to objectively evaluate tic symptoms. The patient or his/her legal representative must given written informed consent before the DBS procedure. If DBS is performed in the context of a trial, the study design has to be approved by the local ethics committee [16].

\section{Treatment refractoriness}

The exact definition of treatment refractoriness in TS has been debated [17]. A significant percentage of patients with TS show spontaneous improvement up to a complete disappearance of most if not all the symptoms during adulthood: DBS is of course indicated for those patients failing to show such improvement of symptoms and related social impairment [18]. The therapeutic strategies used to treat tics and co-morbidities are far from being standardized; although there are many open studies on pharmacotherapy, only a limited number of studies meets rigorous criteria for therapeutic trials. Cochrane reviews and expert views on the pharmacological treatment of TS have recently been published (Fig. 1) [19,20].

Drug therapy often involves the use of antipsychotic agents, which can be associated with a higher risk of adverse effects compared to other drugs. Side effects may persist during adulthood: this fact by itself represent an important element in evaluating refractori- 


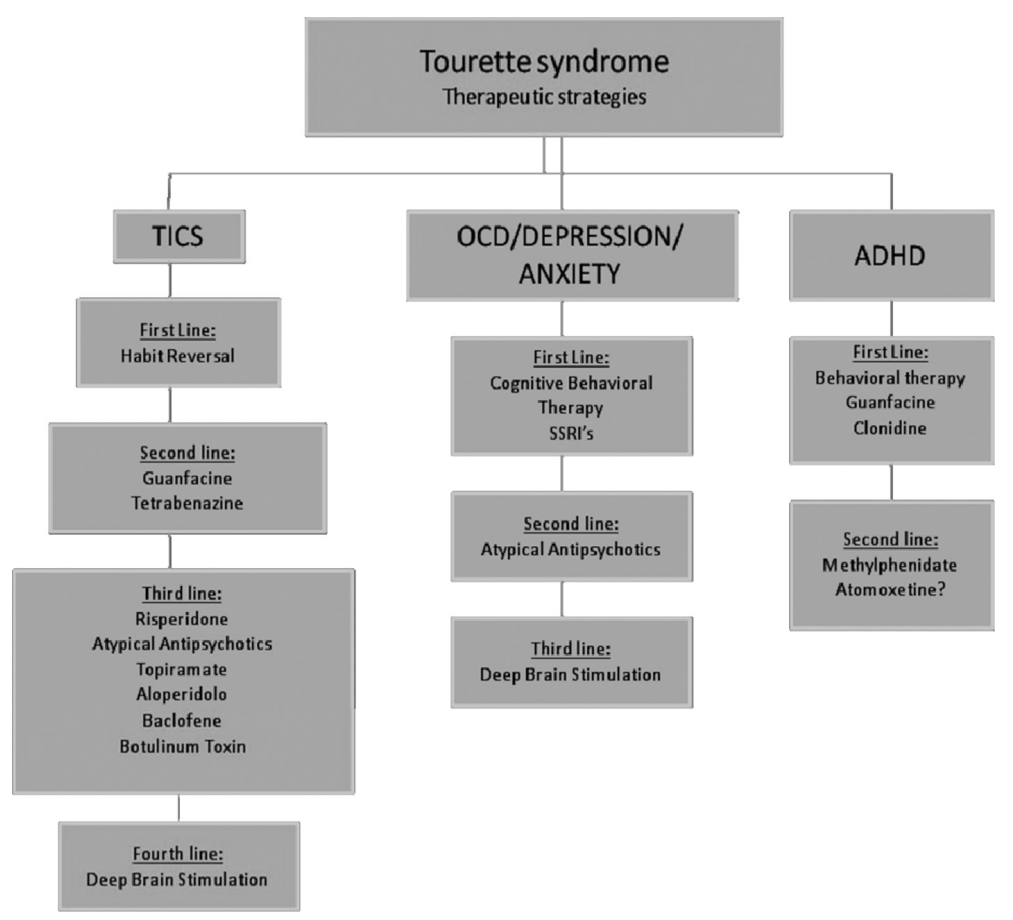

Fig. 1. Proposed algorithm for the treatment of Tourette syndrome, based on the authors' interpretation of evidence-based literature and their own experience (cfr. [20]).

ness. In the scientific literature, drug protocols used before DBS in patients with TS are rarely reported in details [21,22]. Some authors described the use of "maximum dose of established treatments" [23], or "an inadequate response to at least two dopamine blockers or catecholamine depletors" [24], or "failure of best treatment by medication (antipsychotics), or intolerance after a minimum of 6 months of treatment", before DBS [25]. In our department (IRCCS Galeazzi, Milan, Italy), patients with TS are considered treatmentrefractory when they failed to respond to a behavioural intervention, such as habit reversal training, and therapeutic doses of at least three medications of proven efficacy for tics, including antidopaminergic drugs (i.e. haloperidol, pimozide, risperidone, tiapride, sulpiride, aripiprazole), $\alpha-2$ adrenergic agonists (i.e. clonidine and guanfacine), and SSRIs (selective serotonin reuptake inhibitors). Refractoriness is also established by lack of response to innovative drugs, which are often prescribed off label.

\section{Patient age}

Age is an issue of particular relevance for the selection of surgical candidates in TS, since spontaneous remission by the age of 18 has been shown to occur in nearly half of the patients [3]. Performing DBS surgery too early in a patient who can undergo spontaneous improvement means to expose him/her to unnecessary surgical risks, despite the fact that DBS is a mini-invasive, reversible, adjustable procedure. On the other hand, delaying the intervention in patients with severe TS symptoms can expose them to a considerable disadvantage, in terms of quality of life, education, and social inclusion. It is well known that the age from 18 to 25 years is a crucial phase for schooling and vocational qualification, thus the presence of severe tics and comorbidities can lead to far-reaching consequences, including permanent social impairment $[26,27]$. In fact, it is during developmental age that clinical stigmata of the disorder cause the most significant damage, severely and permanently altering the social functioning of the patient, in some cases to such an extent that even after regression of symptoms return to normal social life is almost impossible.

\section{Target choice}

At least eight targets for DBS in patients with TS have been reported. Most studies report experience 
with small case series (less than five). Overall, in this decade DBS for TS has brought more questions than answers. In our department, fifty patients with TS have been treated with DBS. The most targeted area was the ventro-oralis-centromedian-parafascicular nucleus of the thalamus Vo-Cm-Pf (Lat: $5 \mathrm{~mm}$, AP: $-2 \mathrm{~mm}$, $\mathrm{Z}$ : $0 \mathrm{~mm}$ ), with optimal results concerning tics and improvement in OCD and anxiety [28,29]. Stimulation of the nucleus accumbens-anterior limb of the internal capsule (NA-ALIC) was used as first line procedure or as rescue procedure for patients with relevant OCD who failed to respond to Vo-Cm-Pf stimulation. In selected patients with prevalence of dystonic tics, significant reduction of tic severity was obtained targeting the posterior portion of the globus pallidus-pars interna (Gpi). In patients with a clinical picture characterised by significant co-morbidities, we targeted the anterior portion of the GPi [30]. In our opinion, targets should be selected case by case on the basis of the most relevant symptoms (tailoring approach), as different targets seem to have different clinical effects.

\section{Compliance}

Poor patient and caregivers compliance should be considered as a major exclusion criterion. Patient with history of drug addiction have to be carefully evaluated. The role of caregivers in determining outcome of DBS is poorly elucidated. Frequently caregivers may not appreciate amelioration of symptomatology after DBS. The risk is to produce frustration on the patients and decrease the compliance. In most cases physicians focus on patient compliance, while caregiver's compliance is frequently underestimated. From our experience we have learnt that caregiver compliance can be as important as patient compliance. We have had two disposal removals due to caregiver's poor compliance which prevailed over patient will, convincing them of the failure of the surgical procedure. In order to be eligible for DBS, patients should demonstrate compliance with previous treatments [31]. Compliance should be assessed in terms of adherence to pharmacological protocols and psychobehavioural training programs, and attendance to follow-up visits.

\section{Other exclusion criteria}

A controversial issue within exclusion criteria is the finding of brain abnormalities on pre-operative MRI scans. In clinical series of dystonic patients, focal signal abnormalities in the basal ganglia do not contraindicate DBS [32]. On the other hand, in Parkinson disease the presence of chronic ischemic disease or diffuse cerebral atrophy compatible with atypical Parkinsonism represent exclusion criteria for DBS. In our TS series, the presence of vascular abnormalities affected the positioning of the stimulating electrode near the target [33]. There are uncertainties also with regards to general medical conditions representing contraindication to DSB. In various reports, cardiovascular, pulmonary or hematological disorders are considered a contraindication for surgery. In our opinion, patients should be excluded from surgery if they have an American Society of Anesthesiologists (ASA)score of 3 or above which represent an increased risk for general anaesthesia.

Another important aspect related to exclusion criteria is the presence of severe psychiatric co-morbidity. With regards to this, it has to be considered that about $90 \%$ of patients with TS have at least one co-morbid behavioural symptom, such as ADHD, OCD, SIB, impulse dyscontrol and affective dysregulation [2]. The scientific literature is controversial about psychiatric disorders representing exclusion criteria for DBS. For instance, Mink et al. [16] recommend that candidates to DBS must not have co-morbid psychiatric disorders, while Shahed et al. [30] proposed that psychiatric comorbidities in TS should not necessarily represent an exclusion criterion, because it is possible that these behavioural problems will also improve with DBS. It is mandatory to evaluate psychiatric disorder case by case, while previous suicidal attempts should be considered a contraindication.

\section{Conclusion}

Different guidelines on the selection of candidates for DBS in TS have been published to date (Table 1).

From the critical evaluation of these guidelines emerges a substantial difference regarding the minimum age at which DBS might be considered. As stated above, we believe that surgical procedure have to be proposed to younger patients, starting from the age of 18 years. It should also be highlighted that exceptions in terms of younger patients may be considered for DBS when needed, as in the case of the 16-year-old patient reported by Shahed et al. [30]. The second problem concerns the behavioural spectrum of TS, which is referred to as co-morbidity. The phenotype of TS 


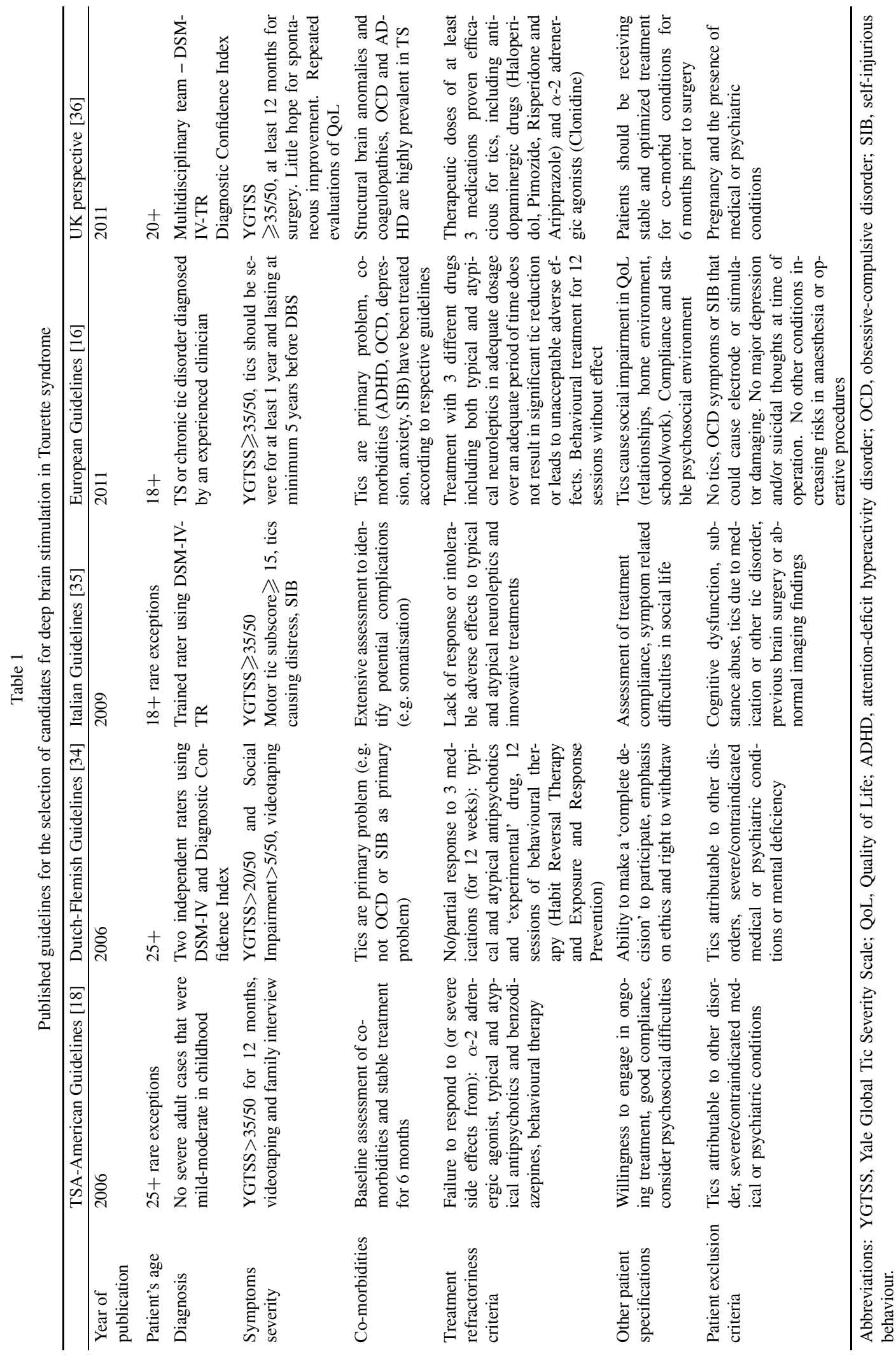


Table 2

Proposed inclusion criteria for deep brain stimulation in Tourette syndrome

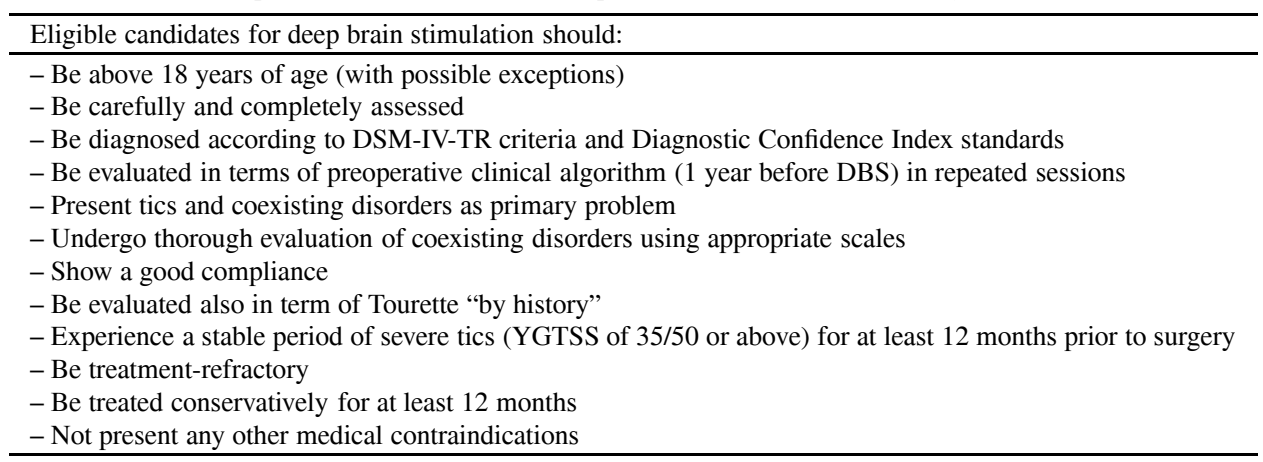

is multifaceted, with special attention to behavioural problems. The issue of whether TS should still be considered as a unitary nosological entity represents a procedural priority. Thus the optimal dissection of TS in each patient is fundamental to understand which patients might benefit from DBS.

In conclusion, TS can no longer be considered a motor disorder and, most importantly, a unitary condition as previously thought. In consideration of this and the data discussed above, we propose the inclusion criteria for DBS used in our department (Table 2), which can be considered a possible platform to establish large multicenter trials to build evidence for DBS protocols and targets in TS.

\section{References}

[1] American Psychiatric Association. Diagnostic and Statistical Manual of Mental Disorders. Fourth Edition, Text Revision (DSM-IV-TR). Washington, DC:APA; 2000.

[2] Cavanna AE, Servo S, Monaco F, Robertson MM. The behavioural spectrum of Gilles de la Tourette syndrome. J Neuropsychiatry Clin Neurosci 2009; 21: 13-23.

[3] Leckman JF, Zhang H, Vitale A, Lahnin F, Lynch K, Bondi $\mathrm{C}$, et al. Course of tic severity in Tourette syndrome: the first decades. Pediatrics 1998; 120: 14-19.

[4] Gross RE, Lozano AM. Advances in neurostimulation for movement disorders. Neurol Res 2000; 22: 247-258.

[5] Lujan JL, Chaturved A, McIntyre CC. Tracking the mechanisms of deep brain stimulation for neuropsychiatric disorders. Front Biosci 2008; 13: 5892-5904.

[6] Hassler R, Dieckmann G. Relief of obsessive-compulsive disorders, phobias and tics by stereotactic coagulations of the rostral intralaminar and medial-thalamic nuclei. In: Laitinen LV, Livingston K, (eds), Surgical approaches in psychiatry. Proceedings of the Third International Congress of Psychosurgery. Cambridge, UK: Garden City Press; 1973; p. 206212.

[7] Porta M, Sassi M, Servello D. Surgical treatment of Tourette. Oxford University Press 2012; in press.

[8] Cheung MY, Shahed J, Jankovic J. Malignant Tourette syndrome. Mov Disord 2007; 22(12): 1743-1750.
[9] Cavanna AE, Schrag A Morley D, Orth M, Robertson MM, Joyce E, Critchley HD, Selai C. The Gilles de la Tourette syndrome-quality of life scale (GTS-QOL): development and validation. Neurology 2008; 71(18): 1410-1416.

[10] Cavanna AE, Critchley HD, Orth M, Stern JS, Young MB, Robertson MM. Dissecting the Gilles de la Tourette spectrum: a factor analytic study on 639 patients. J Neurol Neurosurg Psychiatry 2011; 82(12): 1320-1323.

[11] Cheung MY, Shahed J, Jankovic J. Malignant Tourette syndrome. Mov Disord 2007; 22(12): 1743-1750.

[12] Cavanna AE, David K, Orth M, Robertson MM. Predictors during childhood of future health-related quality of life in adults with Giles de la Tourette syndrome. Eur J Paediatr Neurol 2012; in press.

[13] Jankovic J. Phenomenology and classification of tics. In: Jankovic J (Ed). Tourette syndrome. Neurol Cl N Am, Vol. 15, Philadelphia: WB Saunders, 1997: 267-275.

[14] Kompoliti K, Goetz CG. Clinical rating and quantitative assessment of tics. In: Neurologic Clinics. Jankovic J, (ed.), Tourette syndrome. Philadelphia: WB Saunders, 1997; 15: 239-254.

[15] Bernabei M, Preatoni E, Mendez M, Piccini L, Porta M, Andreoni G. A novel automatic method for monitoring Tourette motor tics through a wearable device. Mov Disord 2010; 25(12): 1967-1972.

[16] Müller-Vahl KR, Cath DC, Cavanna AE, Dehning S, Porta M, Robertson MM, Visser-Vandewalle V, ESSTS Guidelines Group. European clinical guidelines for Tourette syndrome and other tic disorders. Part IV: deep brain stimulation. Eur Child Adolesc Psychiatry 2011; 20(4): 209-217.

[17] Porta M, Sassi M, Menghetti C, Servello D. The need for a proper definition of a "treatment refractoriness" in Tourette syndrome. Front Integr Neurosci 2011; 5: 22.

[18] Mink JW, Walkup J, Frey KA, Como P, Cath D, Delong MR, et al. Patient selection and assessment recommendations for deep brain stimulation in Tourette syndrome. Mov Disord 2006; 21 : 1831-1838.

[19] Verdellen C, van de Griendt J, Hartmann A, Murphy T, ESSTS Guidelines Group. European clinical guidelines for Tourette syndrome and other tic disorders. Part III: behavioural and psychosocial interventions. Eur Child Adolesc Psychiatry 2011; 20(4): 197-207.

[20] Jankovic J, Kurlan R. Tourette syndrome: Evolving Concepts. Mov Disord 2011; 26(6): 1149-1156.

[21] Panagiotopoulos C, Ronsley R, Elbe D, Davidson J, Smith DH. First do no harm: promoting an evidence-based approach 
to atypical antipsychotic use in children and adolescents. J Can Acad Child Adolesc Psychiatry 2010; 19: 124-137.

[22] Pringsheim T, Pearce M. Complications of antipsychotic therapy in children with Tourette syndrome. Pediatr Neurol 2010; 43(1): 17-20.

[23] Kuhn J, Lenartz D, Mai JK, Huff W, Lee SH, Koulousakis A, Klosterkoetter J, Sturm V. Deep brain stimulation of the nucleus accumbens and the internal capsule in therapeutically refractory Tourette-syndrome. J Neurol 2007; 254(7): 963965.

[24] Maciunas RJ, Maddux BN, Riley DE, Whitney CM, Schoenberg MR, Ogrocki PJ, et al. Prospective randomized doubleblind trial of bilateral thalamic deep brain stimulation in adults with Tourette syndrome. J Neurosurg 2007; 107: 1004-1014.

[25] Welter ML, Mallet L, Houeto JL, Karachi C, Czernecki V, Cornu $\mathrm{P}$, et al. Internal pallidal and thalamic stimulation in patients with Tourette syndrome. Arch Neurol 2008; 56: 952957.

[26] Eddy CM, Cavanna AE, Gulisano M, Agodi A, Barchitta M, Calì P, Robertson MM, Rizzo R. Clinical correlates of quality of life in Tourette syndrome. Mov Disord 2011; 26: 735-738.

[27] Eddy CM, Rizzo R, Gulisano M, Agodi A, Barchitta M, Calì P, Robertson MM, Cavanna AE. Quality of life in young people with Tourette syndrome: A controlled study. J Neurol 2011; 258: 291-301.

[28] Servello D, Sassi M, Brambilla A, Porta M, Haq I, Foote KD, Okun MS. De novo and rescue DBS leads for refractory Tourette syndrome patients with severe comorbid OCD: a multiple case report. J Neurol 2009; 256(9): 1533-1539.

[29] Sassi M, Porta M, Servello D. Deep brain stimulation therapy for treatment-refractory Tourette's Syndrome: A review. Acta Neurochir (Wien) 2011; 153(3): 639-645.

[30] Shahed J, Poysky J, Kenney C, Simpson R, Jankovic J. GPi deep brain stimulation for Tourette syndrome improves tics and psychiatric comorbidities. Neurology 2007; 68: 159-160.

[31] Eddy CM, Rickards HE, Cavanna AE. The cognitive impact of antiepileptic drugs. Ther Adv Neurol Dis 2011; 4: 380-402.

[32] Gavarini S, Vayssière N, Delort P, Cif L, Biolsi B, Tancu C, Vasques X, Plagnol S, Bonafe A, Coubes P. Stereotactic MRI in DYT1 dystonia: Focal signal abnormalities in the basal ganglia do not contraindicate deep brain stimulation. Stereotact Funct Neurosurg 2008; 86(4): 245-252.

[33] Servello D, Sassi M, Brambilla A, Defendi S, Porta M. LongTerm, Post-Deep Brain Stimulation Management of a Series of 36 Patients affected with refractory Gilles de la Tourette syndrome. Neuromodulation 2010; 13: 187-194.

[34] Ackermans L, Temel Y, Cath D, van der Linden C, Bruggeman R, Kleijer M, Nederveen P, Schruers K, Colle H, Tijssen MA, Visser-Vandewalle V, Dutch Flemish Tourette Surgery Study Group. Deep brain stimulation in Tourette's syndrome: Two targets? Mov Disord 2006; 21(5): 709-713.

[35] Porta M, Servello D, Sassi M, Brambilla A, Defendi S, Priori A, Robertson M. Issues related to deep brain stimulation for treatment refractory Tourette syndrome. Eur Neurol 2009; 62: 264-273.

[36] Cavanna AE, Eddy CM, Mitchell R, Pall H, Mitchell I, Zrinzo $\mathrm{L}$, et al. An approach to deep brain stimulation for severe treatment-refractory Tourette syndrome: the UK perspective. Br J Neurosurg 2011; 25(1): 38-44. 


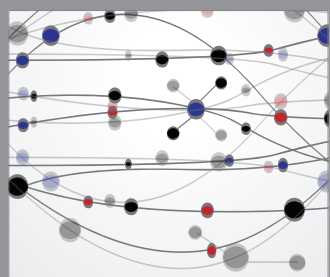

The Scientific World Journal
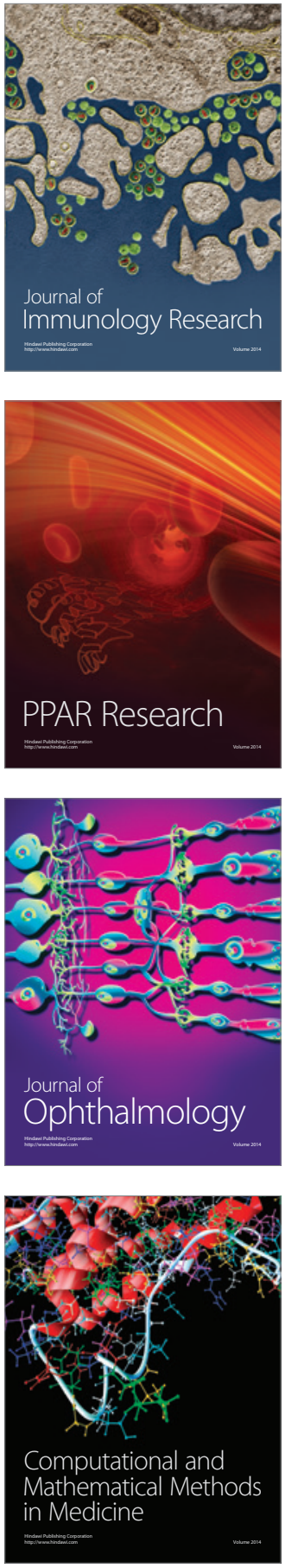

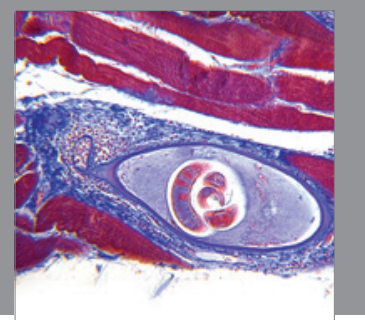

Gastroenterology

Research and Practice
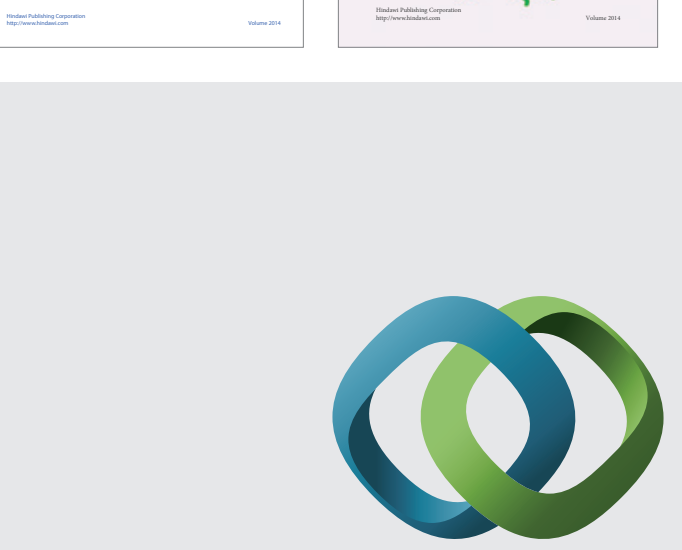

\section{Hindawi}

Submit your manuscripts at

http://www.hindawi.com
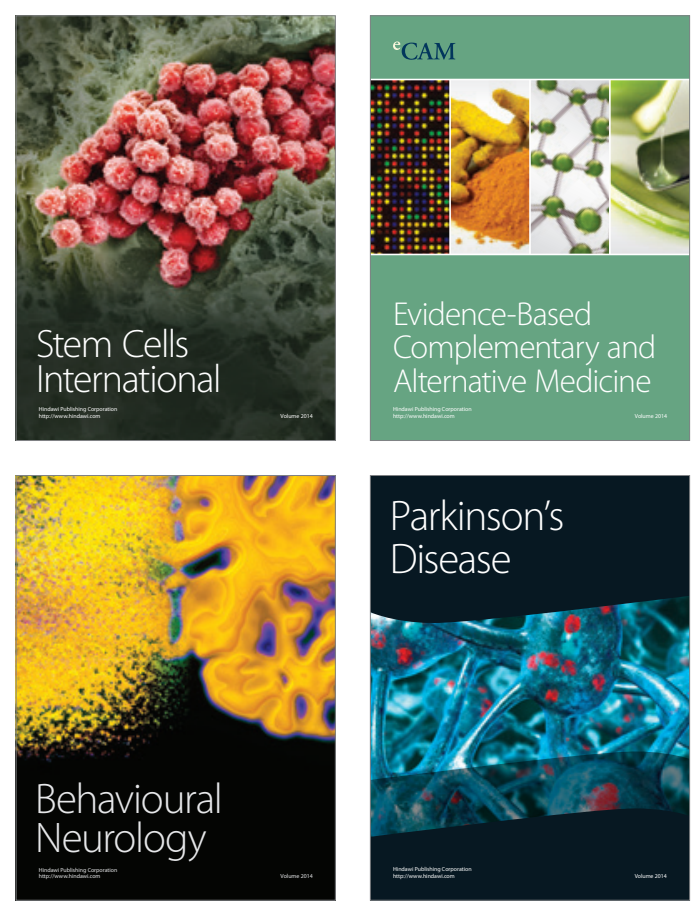

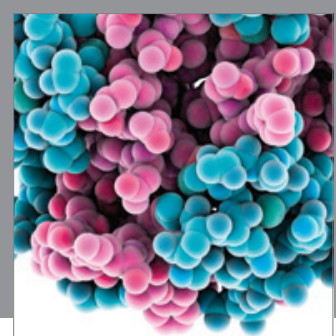

Journal of
Diabetes Research

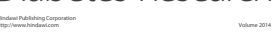

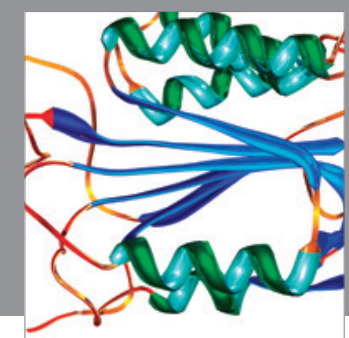

Disease Markers
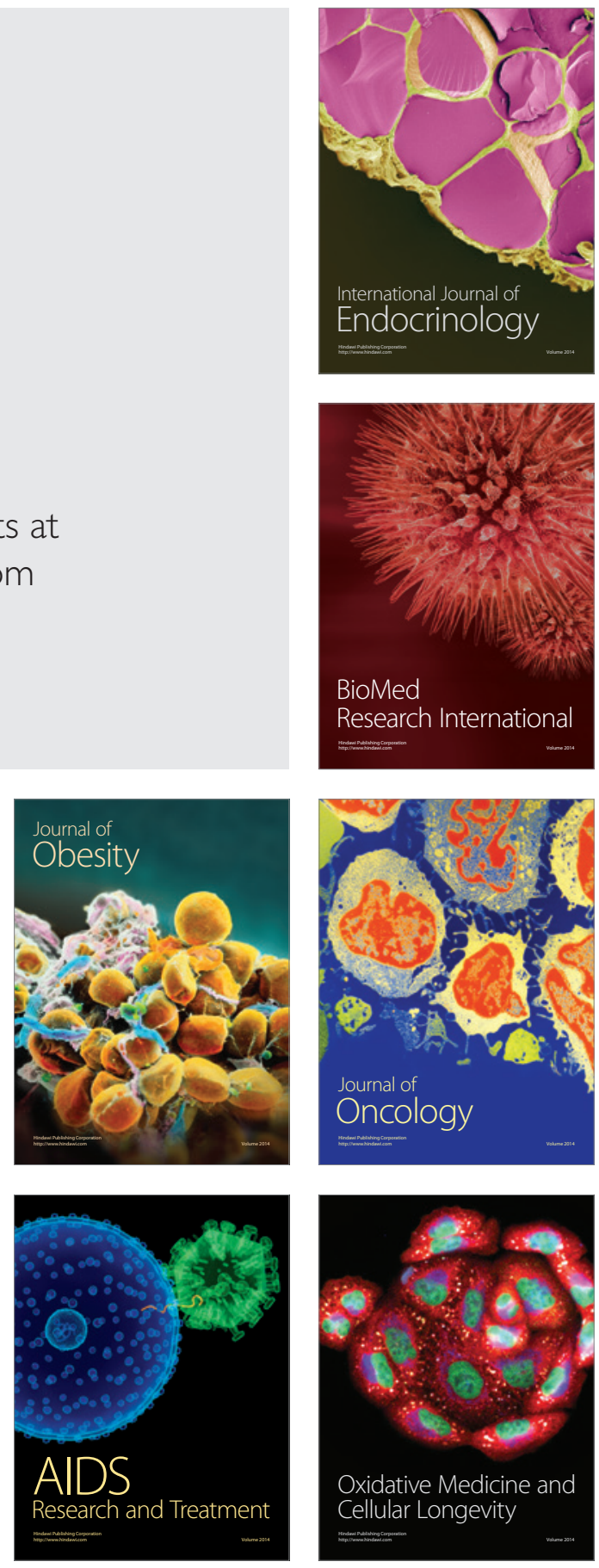